\title{
Calculation of magnetic field strength for coil system designed to free from magnetic force and to confine the high $\beta$ plasma
}

\author{
無電磁力・高 $\beta$ コイルの磁場強度の計算
}

\author{
Katsuhiko SUNAKO, Takeshi YOSHIDA, Akihiro MATSUBARA, Akira TONEGAWA, and \\ Kenzo NANRI \\ Department of Physics, Tokai University \\ 1117, Kitakaname, Hiratsuka, 259-12 JAPAN \\ TEL ; 81-463-58-1211, FAX ; 81-463-58-1203
}

Kazutaka KAWAMURA, Eiji YABE, and Kazuo TAKAYAMA

Institute of Research and Development, Tokai University,

1117, Kitakaname, Hiratsuka, 259-12 JAPAN

TEL ; 81-463-58-1211, FAX ; 81-463-58-1203

Teruo YAJIMA, and Tomohiro MIYATA

Department of Nuclear Engineering, Tokai University

1117, Kitakaname, Hiratsuka, 259-12 JAPAN

TEL ; 81-463-58-1211, FAX ; 81-463-58-1203

( Received 19, May 1995 Accepted 14, August 1995 )

\begin{abstract}
The coil system designed to free from the magnetic force and to confine the high $\beta$ plasma is proposed. We carry out the calculation of the magnetic field for the proposed coil by using the Biot-Savart law. It is noted that no or very weak magnetic fields are appeared in the central region of the proposed coil, while the strong magnetic field localizes at the outside of this region, being satisfied the requirement of confining the high $\beta$ plasma.

The simplified model is considered in order to reduce the cpu time for the computation. From the results of the computation, it is shown that the proposed coil is able to be replaced by the simplified model.
\end{abstract}

Key words : magnetic field strength, Biot-Savart law, coil system designed to free from force, high $\beta$ plasma, proposed coil, simplified model, cpu time

\section{I .INTRODUCTION}

The magnetic confinement of the plasma has been used extensively for the developmental research of the nuclear fusion. The type of the coil wound with wires is required to be free from the strong magnetic force and to provide the high $\beta$ (the ratio of the plasma kinetic pressure to the magnetic pressure). ${ }^{1}$ One of the possible way to meet the requirements mentioned above is that the wires cross perpendicularly each other and the magnetic field at the confined region of plasma, called as the center of plasma, tends to almost zero while the magnetic field to be produced at the outside of this region is kept as high as possible.

A proposed coil is shown in Fig.1. As indicated from this figure, two wires intersected perpendicularly are wound arourd the cylinder diagonally.

On the other hand, the proposed coil is able to be replaced by a simplified model as shown in Fig.2, in which the wires are placed parallel with the principal axis of the cylinder. The calculation of the magnetic field of the simplified model is rather facilitated, in contrast with that of the proposed coil. It is, thus , expected that the central processing unit (cpu) time for simplified model is much shorter than that for proposed coil.

The present work will show that calculated magnetic field for proposed coil satisfies the requirement of confining the high $\beta$ plasma and how the computational results for simplified model provides the same results as the computation for proposed coil gives.

\section{II.ASSUMPTIONS AND COMPUTATIONAL PROCEDURE}

The coordinate axes, $x, y$, and $z$, in an orthogonal coordinate used for present computation is also shown in Fig. 1. 


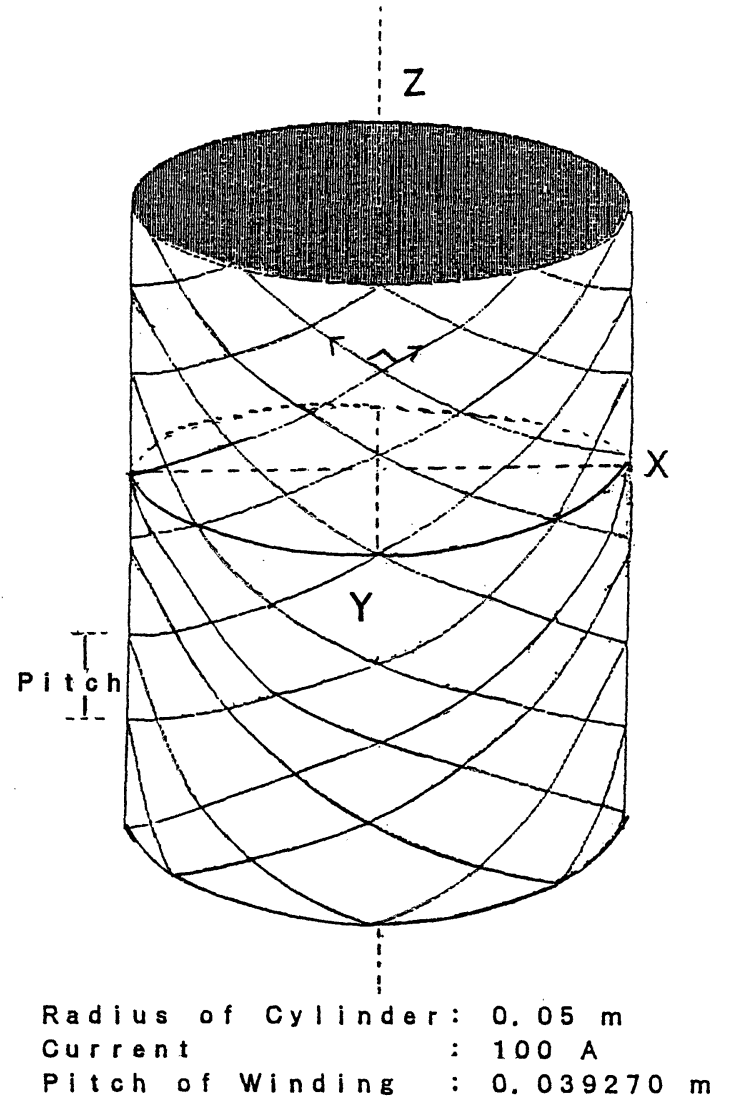

Fig.1. Proposed coil.

The magnetic field for the proposed coil was calculated with a help of the Biot-Savart law. The equation expressed in vector form is as follows;

$$
\mathrm{d} \mathbf{H}=\mathrm{I} /\left(4 \pi \mathrm{r}^{3}\right) \mathrm{d} \mathbf{s} \times \mathbf{r}
$$

where $\mathrm{dH}$ is the strength of the magnetic field at a selected point $(\mathrm{A} / \mathrm{m})$, I the electric current $(\mathrm{A})$, $\mathrm{d} S$ an element of the wire $(\mathrm{m})$ and $\mathbf{r}$ a distance between $\mathrm{ds}$ and the selected point $(\mathrm{m})$. A truncation error of the calculated magnetic field for proposed coil amounted to as low as 1 per cent.

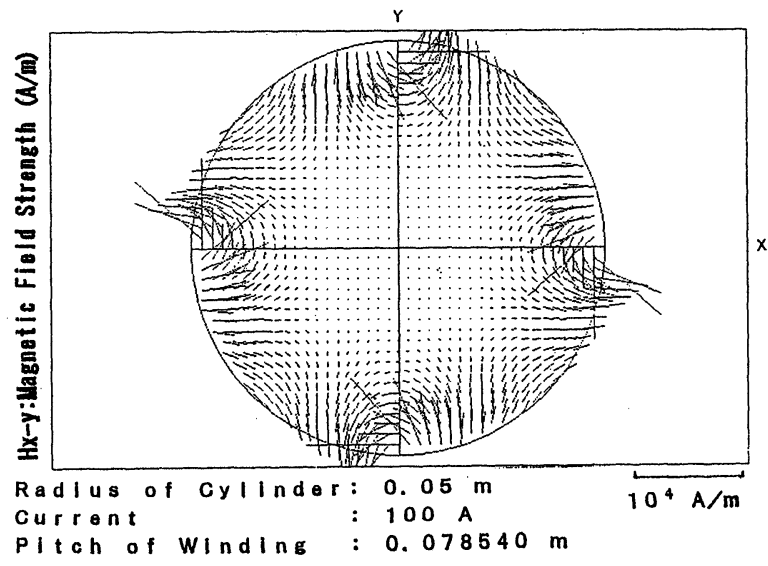

Fig.3a. Calculated magnetic field at the $x-y$ plane for proposed coil.

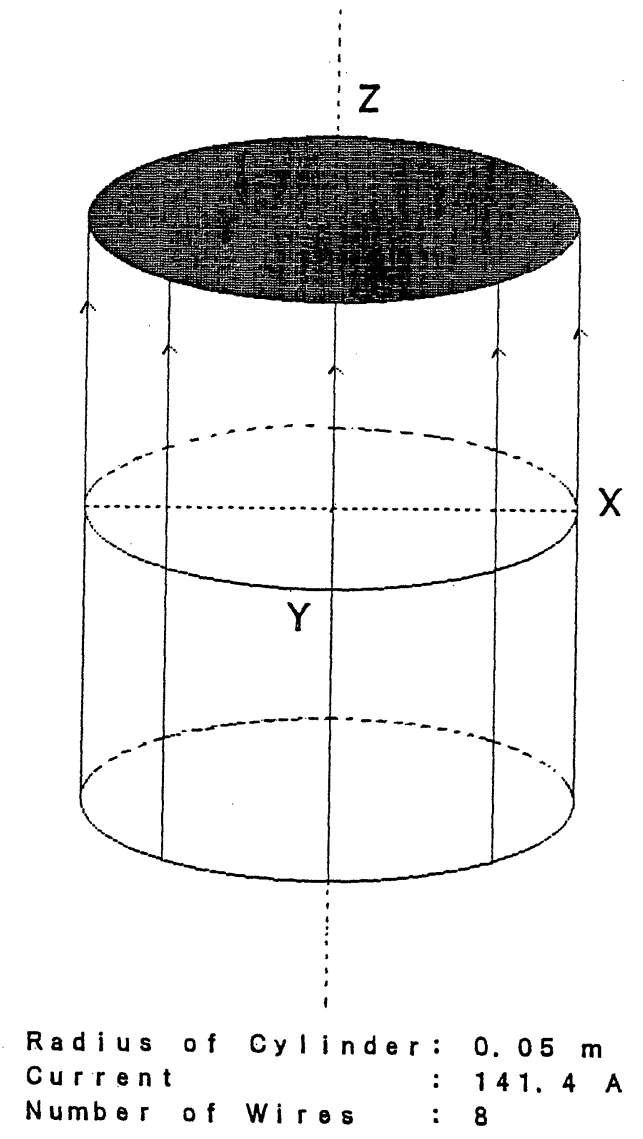

Fig.2. Simplified model.

In contrast with the calculation of magnetic field for proposed coil, that for simplified model is computed by the following equation;

$\mathrm{H}=\mathrm{I} /\left(2 \pi \mathrm{r}^{\prime}\right)$

where $r^{\prime}$ is a radius of the cylinder $(\mathrm{m})$. In the case of the simplified model, the length of wire was assumed to be infinite.

The computation was carried out by using the FORTRAN and the graphic display was achieved by the $\mathrm{C}$ programming language.

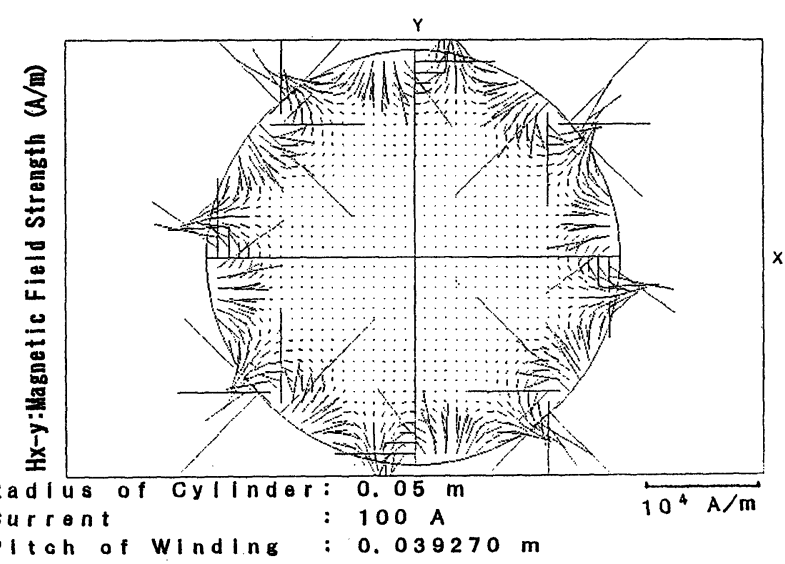

Fig.3b. Calculated magnetic field at the $x-y$ plane for proposed coil. 


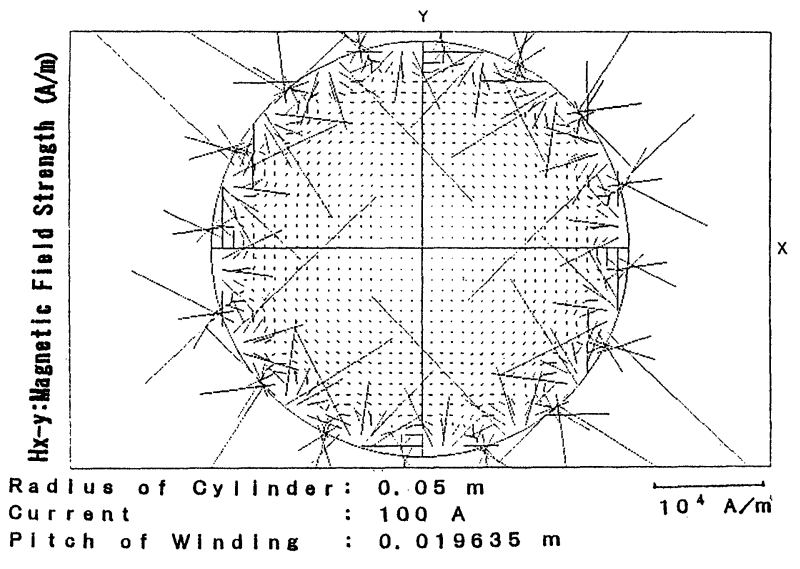

Fig.3c. Calculated magnetic field at the $x-y$ plane for proposed coil.

The values used for the calculation were as follows; The electric currents flowed through the wires for proposed coil and for simplified model were 100 $\mathrm{A}$ and $141.4 \mathrm{~A}(=100 \mathrm{x} \sqrt{2} \mathrm{~A})$, respectively, and the radius of cylinder was $0.05 \mathrm{~m}$.

\section{III.RESULTS AND DISCUSSION}

The calculated magnetic field at the selected $\mathrm{x}$ y plane for proposed coil in which four, eight, and sixteen cross points exist are shown in Figs. 3(a), 3(b), and $3(\mathrm{c})$, respectively. Those at the $x-y$ plane for simplified model in which four, eight, and sixteen wires locate on the circumference of the cylinder are also illustrated in Figs. 4(a), 4(b), and 4(c), respectively. From these figures, it is noted that no or very weak magnetic fields are appeared in the central region of the cylinder, i.e., confined region of plasma, while the strong magnetic fields localize at the outside of this region, being satisfied the requirement of confining the high $\beta$ plasma.

On the other hand, it is stressed that the magnetic fields in the $z$ direction become to be nil both for proposed coil and for simplified model. An example of the calculated magnetic field in the $z$

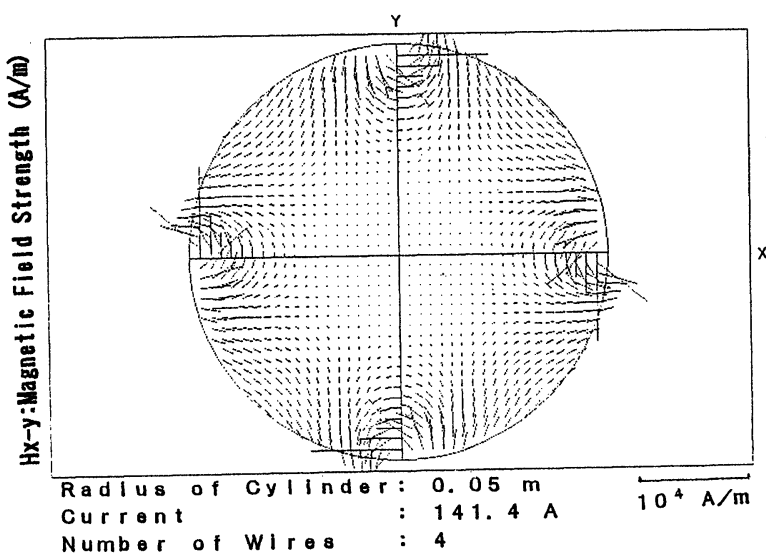

Fig.4a. Calculated magnetic field at the $x-y$ plane for simplified model.

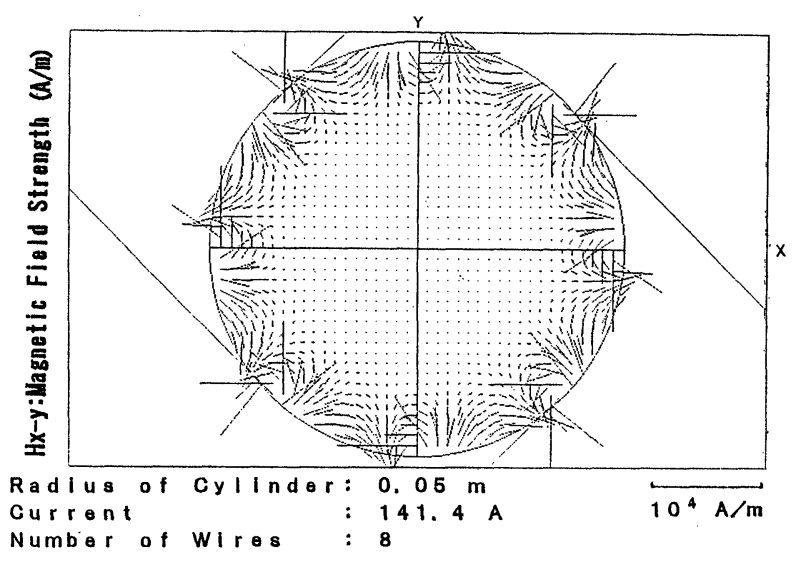

Fig.4b. Calculated magnetic field at the $x-y$ plane for simplified model.

direction for proposed coil is shown in Fig.5.

A glance at figures 3 and 4 shows that the cusp-like magnetic field is appeared at the places between two cross points for proposed coil or between two wires for simplified model. The presence of such cusp field is supported by an experiment. ${ }^{2}$

It is evident from figures 3 and 4 that the feature and the strength of the magnetic field in the $x-y$ plane for proposed coil are more likely to be similar to those for simplified model. We may, therefore, speculate that the proposed coil is able to be replaced by the simplified model.

The cpu time for the simplified model with 8 wires amounts to about one over two hundred as short as those for proposed coil with 8 cross points in the selected plane. In the further case where the wires for simplified model or the cross points in selected plane for proposed coil increase, the simplified model clearly favors the cpu time, compared with that for proposed coil.

As for the separatrix for proposed coil or for simplified model, it is expected to draw schematically as indicated in Fig. 6.

The results presented in this paper will be used

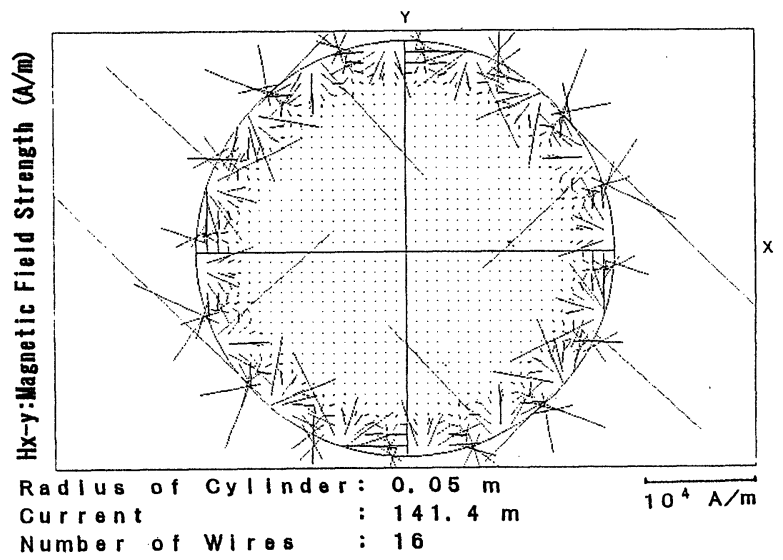

Fig.4c. Calculated magnetic field at the $x-y$ plane for simplified model. 


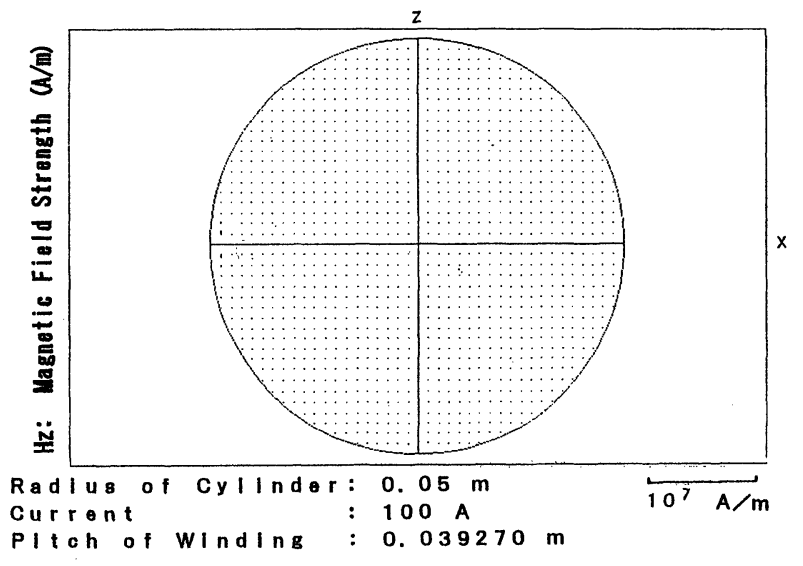

Fig.5. Calculated magnetic field in the $\mathrm{z}$ direction for proposed coil.

in a subsequent paper.

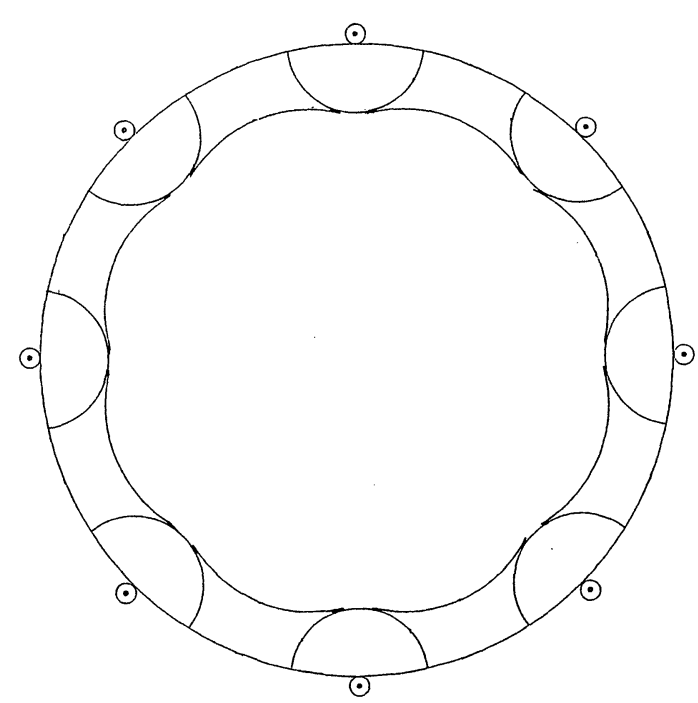

Fig.6. Separatrix both for propsed coil and for simplified model.

\section{IV.CONCLUSION}

In view of the effective confinement of plasma, the coil which meets the requirements, such as the coil being free from the strong magnetic force and having the confinement of the high $\beta$ plasma, is desired to design. The requirement freed from magnetic force for proposed coil can be achieved by the wires crossed perpendicularly each other. The magnetic field is computed numerically by using the Biot-Savart law for proposed coil. It is noted that no or very weak magnetic fields are appeared in the central region of the proposed coil, while the strong magnetic field localizes at the outside of this region, being satisfied the requirement of confining the high $\beta$ plasma.

On the other hand, the simplified model which is equivalent to the proposed coil is considered in order to reduce the cpu time for the computation. The feature and the strength of the magnetic field in the $x-y$ plane for proposed coil are more likely to be similar to those for simplified model. It is evident that the proposed coil is able to be replaced by the simplified model.

The cpu time for proposed coil is much shorter than that for the proposed coil.

The results mentioned in present paper will be used in a subsequent paper.

The authors wish to express their gratitude for the finantial assistance both from the Shinsei Foundation and from Grant-in-Aid for General Scientific Research (C), No. 06680471, the Ministry of Education, Science, Sports and Culture.

\section{REFERENCES}

${ }^{1}$ D. A. Frank-Kamenetskii, Plasma : The Fouth State Matter (Plenum Press. New York 1972), p.48.

${ }^{2}$ T. Miyata, K. Kawamura and K. Takayama, to be submitted. 